\title{
The Effect of Cytomegalovirus Reactivation on Relapse in Acute Myeloblastic Leukemia after Allogeneic Hematopoietic Stem Cell Transplantation
}

\author{
Yeral $\mathbf{M}^{*}$ and Aytan $\mathbf{P}$ \\ Department of Hematology, Faculty of Medicine, Baskent \\ University, Turkey \\ *Corresponding author: Mahmut Yeral, Department \\ of Hematology, Baskent University Adana Dr.Turgut \\ Noyan Teaching and Medical Research Center, Yüregir \\ Adana, Turkey
}

Received: September 21, 2017; Accepted: October 16, 2017; Published: October 30, 2017

\section{Editorial}

Cytomegalovirus (CMV) infection is one of the most significant causes of non-relapse mortality after allogeneic peripheral stem cell transplantation (PSCT). However, monitoring for CMV and preemptive treatment approaches have reduced this rate considerably. A few studies have even reported that CMV viremia can increase the graft versus leukemia effect $[1,2]$.

We investigated the effects of CMV reactivation on disease free survival in patients with acute myeloblastic leukemia (AML) in the first 100 days after allogeneic PSCT.

\section{Patients and Methods}

Patients who were diagnosed with AML and underwent allogeneic PSCT at Baskent University, Research and Application Hospital, were included in this study. Patients who scored two or higher on the ECOG scale, were in full remission, had a HLA compatible sibling donor, or were being administered a myeloablative conditioning regimen were included.

Patients on non-myeloablative conditioning regimens, who had unrelated allogeneic PSCT, who (or their donors) showed CMV seronegativity before transplantation, or who developed relapse or nonrelapse mortality in the first 100 days after transplantation were excluded.

Among 69 patients who met the criteria, 30 were female and 39 were male with a median age of 49 years (range, 18-63). The conditioning regimen included total dosages of $150 \mathrm{mg} / \mathrm{m}^{2}$ Fludarabine, 9.6$12.8 \mathrm{mg} / \mathrm{kg}$ Busulfan and 15-30 mg/kg ATG. All patients received immunosuppressive therapy of cyclosporine and short term MTX. Median days to neutrophil and platelet engraftment were 12 (range 10-16) and 14 (range 11-18), respectively. A prophylaxis of fluconazole and acyclovir was administered until the second month of treatment, and trimethoprim/sulfamethoxazole was administered during the period of immunosuppressive therapy. Following the day of engraftment, nucleic acid samples from the plasma of the patients were used to quantify CMV DNA copy numbers by RTPCR.
We investigated CMV reactivation weekly in the first month, biweekly in the second month, and then monthly until month 6; 1000 copies/mL was considered the threshold value. Notably, this value was reached in 20 patients (29\%). Ganciclovir $(10 \mathrm{mg} / \mathrm{kg} /$ day $)$ was administered to $80 \%$ of the patients, none of whom developed resistance to the therapy. There were no statistically significant differences between the two groups with respect to age, sex, and risk group. Median disease free survival was 45 months (range, 463) in CMV positive patients and 26 months (range, 465) in CMV negative patients. Two year disease free survival was $75 \%$ in CMV positive patients and 53\% in CMV negative patients. There was a statistically significant difference between the two groups in terms of disease free survival $(\mathrm{p}<0.001)$.

\section{Discussion and Results}

CMV is a common herpes virus, and CMV replication commonly occurs in immunosuppressed patients. However, the development of symptomatic infection is associated with a high mortality rate and is a major cause of nonrelapse mortality in patients receiving allogeneic PSCT. Therefore, a preemptive approach to treatment is now preferred.

Early CMV peptide replication has been linked to defective $\mathrm{T}$ cell function, leading to increased relapse rates [3]. Conversely, natural killer cells may become activated during CMV viremia, leading to an indirect increase in the antileukemic response. Another possible mechanism could be that blasts express CMV antigens, and therefore active $\mathrm{T}$ cells specific to CMV have cytotoxic effects on the blasts $[1,4]$. In a retrospective study, Elmaagacli, et al. compared CMV reactivation and survival in 266 patients with AML after allogeneic PSCT. The cumulative relapse rate in patients with CMV reactivation during the early transplantation period was $9 \%$, whereas that in those without reactivation was $42 \%$ [2]. Similarly, in our study, CMV reactivation had a positive impact on disease free survival. Antiviral agents used during the preemptive approach and symptomatic infections may sometimes cause serious morbidity and mortality via cytopenia, graft loss, and nephrotoxicity. Steroids and other immunosuppressive agents used to treat cases of graft versus host disease may contribute to the development of opportunistic infections. Taking all of this consideration, patients should be closely monitored and given support as required as well as prophylaxis against opportunistic pathogens.

\section{References}

1. Foley B, Cooley S, Verneris MR, Pitt M, Curtsinger J, Luo X, et al. Cytomegalovirus reactivation after allogeneic transplantation promotes a lasting increase in educated NKG2C+ natural killer cells with potent function. Blood. 2012; 119: 2665-2674.

2. Elmaagacli $A H$, Steckel NK, Koldehoff $M$, Hegerfeldt $Y$, Trenschel R, 
Ditschkowski $M$, et al. Early human cytomegalovirus replication after transplantation is associated with a decreased relapse risk: evidence for a putative virusversusleukemia effect in acute myeloid leukemia patients. Blood. 2011; 118: 1402-1412.

3. Nakamura R, Battiwalla M, Solomon S, Follmann D, Chakrabarti S, Cortez K, et al. Persisting posttransplantation cytomegalovirus antigenemia correlates with poor lymphocyte proliferation to cytomegalovirus antigen and predicts for increased late relapse and treatment failure. Biol Blood Marrow Transplant. 2004; 10: 49-57.

4. Barrett AJ. CMV: when bad viruses turn good. Blood. 2011; 118: 1193-1194.
Ann Hematol Oncol - Volume 4 Issue 10 - 2017

ISSN : 2375-7965 | www.austinpublishing group.com

Yeral et al. (C) All rights are reserved
Citation: Yeral M and Aytan P. The Effect of Cytomegalovirus Reactivation on Relapse in Acute Myeloblastic Leukemia after Allogeneic Hematopoietic Stem Cell Transplantation. Ann Hematol Oncol. 2017; 4(10): 1175. 